\title{
Modeling Of Decision Maker under Imperfect Information
}

\author{
Author Details: Latafat A. Gardashova \\ Department of Computer-Aided Control Systems \\ Azerbaijan State Oil and Industry University \\ Baku, Azerbaijan Republic
}

Abstract - In real-life, imperfect information is commonly present in all the components of the decision making problem. In decision making problems a DM is almost never provided with perfect, that is ideal decision relevant information to determine states of nature, outcomes, probabilities, etc. We are known that, relevant information almost always comes imperfect. Imperfect information is information which in one or more respects is imprecise, uncertain, incomplete, unreliable, vague or partially true [1]. Imprecision is one of the widest concepts including variety of cases. We will discuss uncertainty concepts of imperfect information and it application to problem modeling of decision maker. In the first stage of the modeling the identification determinants of a decision maker was implemented using Delphi method. The aim of the second stage consists of the linguistic evaluation of the factors. At the final stages decision makers model was realised by using possibilityprobability based method and Dempster-Shafer theory based model.

\section{INTRODUCTION}

Two main concepts of imperfect information are imprecision and uncertainty[1,2]. For purposes of differentiation between imprecision and uncertainty, Prof. L.A. Zadeh suggested the following example: "For purposes of differentiation it is convenient to use an example which involves ethnicity. Assume that Robert's father is German and his mother's parents were German and French. Thus, Robert is 3/4 German and 1/4 French. Suppose that someone asks me: What is Robert's ethnicity. If my answer is: Robert is German, my answer is imprecise or, equivalently, partially true. More specifically, the truth value of my answer is 3/4. No uncertainty is involved. Next, assume that Robert is either German or French, and that I am uncertain about his ethnicity. Based on whatever information I have, my perception of the likelihood that Robert is German is 3/4. In this case, $3 / 4$ is my subjective probability that Robert is German. No partiality of truth is involved. No partiality of truth is involved. A proposition is a carrier of information. In the above example, call it the Robert example, the information carried by the proposition "Robert is German" is precise but not entirely correct, that is, is partially true. When imprecision is related to partiality of truth with no uncertainty involved, it will be referred to as strict imprecision, or s-imprecision for short. As was noted already, there is no connection between s-imprecision and uncertainty."

In this work we will discuss modelling of decision maker under imperfect information.

Making decisions is certainly the most important task of a manager and it is often a very difficult one. It depends on two factors: the statement of the decision making problem and the determinants of a decision maker. Decisions are an inevitable part of human activities. It requires the right attitude. Every problem properly perceived, becomes an opportunity. In most cases the decision maker must view the problems as opportunities rather than solving problems. A pessimist sees the difficulty in every opportunity, an optimist sees the opportunity in every difficulty. It all depends on the decision-maker's attitude. Decision maker looks at problems using reaction and emotion. Decision making depends on a character of a decision maker. This requires including different behavioral characteristics of decision maker into decision making model.

The analysis of the existing works [3-15] of the field modeling of decision maker shows that emotion, altruism, reciprocity, fairness, social responsibility and etc. are basic attributes of human behavior. Authors of works [3-7] developed theory of reciprocal altruism for games behavior. Decision maker modeling under second-order uncertainty using the method based on the possibility-probability measure is discussed in work [16,19.20].

[21] is devoted to the problems of decision making in fuzzy environment for management systems of oil 
refinery enterprise (ORE), namely, to the problems of development of new methods and tools which allow to take into account uncertainty of environment and a decision maker's behavior (DM). Two main factors characterized by uncertainty and influencing decision making in management systems of ORE are determined and necessity of development of new methods of decision making under second-order uncertainty is argued.

It is conducted an analysis of psychological determinants of a DM which influence decisions made under uncertainty with the latter intrinsic for both a DM's behavior and a decision making environment. On the base of the suggested analysis, DM's behavior has been modeled by using of fuzzy measure, possibility measure and belief measure.

Human behavioral modeling based on the Dempster -Shafer theory of belief and fuzzy logic is suggested by Yager in [17]. In [17] authors considers the appropriateness of fuzzy sets and fuzzy production rules for representing human centered cognitive concepts. It is noted that production systems is one of the oldest techniques of knowledge representation. Human behavioral modeling requires an ability to formally represent experienced informative or cognitive concepts that are often at best described in imprecise linguistic terms. It is shown in [17] how probabilistic uncertainty can be included into the output of a fuzzy rule by using Dempster-Shafer paradigm. This methodology that combines fuzzy and probabilistic uncertainty provides a framework for creation of models that can include both the concepts and unpredictability needed to model human behavior.

A tractable model of reciprocity and fairness is discussed in [6]. The income distribution and the kindness or unkindness of others' choices systematically affect a person's emotional state. The emotional state systematically affects the marginal rate of substitution between own and others' payoffs, and thus the person's subsequent choices. The proposed model is applied to two sets of laboratory data: simple binary choice mini-ultimatum games, and Stackelberg duopoly games with a range of choices. The results confirm that other-regarding preferences respond to others' intentions as well as to the income distribution. Different approaches to decision making problems to introducing trust, reciprocity, altruism, fairness etc were proposed by James C.Cox and his colleagues and other authors[22-30].

The existing approaches don't deal with possibilistic and probabilistic uncertainty which is characterizing decision makers behavior.

In this paper we try to do modeling of decision maker by using emotion and altruism factors.

The rest of the paper is organized as follows: In section 2 the process of determining decision makers attributes and a statement of the problem are given.

In section 3 modeling process is shortly described under second-order uncertainty using the possibilityprobability measure based method.

In section 4 we create the decision makers model by using obtained data .

Decision maker behavioral modelling using fuzzy and Dempster-Shafer theories suggested in 5. Section 6 is conclusion.

\section{Statement Of The Problem}

The basic problem is to evaluate personal quality of a decision maker by using psychological determinants.

For determining psychological determinants as basic factors influencing a choice of a decision maker we use the Delphi method. For determining basic factors of a decision maker the following questionnaire is created:

\section{Query 1.}

Please indicate by "+" which of the following should be considered as determinants of a decision maker (see in table 1).

Query 2. Identification of total index of a DM.

Please indicate what term should be used for a total index (resulting dimension) of a DM as an overall evaluation to be determined on the base of the determinants indicated in the previous query. 
TABLE I

\section{Determinants OF A DECision MAKER}

\begin{tabular}{|l|l|}
\hline Factor & Mark \\
\hline Trust & \\
\hline Altruism & \\
\hline Reciprocity & \\
\hline Emotion & \\
\hline Risk & \\
\hline Social responsibility & \\
\hline Tolerance to ambiguity & \\
\hline Add new factor if necessary & \\
\hline ....... & \\
\hline ....... & \\
\hline
\end{tabular}

A) personal quality

B) power of decision

C) other(please indicate)

These surveys have been sent the Internet to experts. The answers received from experts(see Fig.1) are operated on the basis of Delphi method.

Altruism, emotion, trust, reciprocity, risk, social responsibility, tolerance to ambiguity etc. are obtained as the basic determinants. Therefore in this work two psychological determinants are chosen for modelling of the decision-maker[21].

As the index decision-making of the decision-maker personal quality is accepted. The following type model is offered on the basis of received answers:

IF $\mathrm{U}_{-} 1$ is $A \_i 1$ and $\mathrm{U} \_2$ is $A \_i 2$ and $\mathrm{U} \_\mathrm{r}$ is $A \_i r$ THEN $\mathrm{V}$ is $\mathrm{Di}$ and $C F i \in] 0 ; 100]$

where $\mathrm{CFi}$ - is the confidence degree of the rule that is defined by expert. It expresses the belief degree of the expert to the truth degree of the rule. $A \_i 1, A \_i 2$, $A \_i r, D i$ are linguistic value of the linguistic variable U_1,U_2, U_r, V.

\section{MODELING OF A DECISION MAKER UNDER SECOND-ORDER UNCERTAINTY USING THE Possibility-Probability Measure BaSED METHOD}

Knowledge in a production systems can be described in different ways. Some of the post-modern techniques for representation of knowledge include logical calculus, production systems and structured model. This work is devoted to the production system based approaches of knowledge representation. The production systems is the simplest. A production systems consist of three items:1) a set of production rules,2) dynamic database, called the working memory, 3) control structure or interpreter, which interprets the database using the set of production rules. The production system has large applications in decision making problems, in oil refinery problem, in psychology, in business problems, in technical problems, in social sciences[3,21,31].

The structure of a production rule can be formally stated as follows. Before presenting the technique for knowledge representation by product systems, we define the term knowledge, which the widely used in this paper.

The production description of knowledge in the knowledge base of decision maker is based on fuzzy interpretation of antecedents and consequents in production rules[3].

$R^{k}: I F x_{1}$ is $\tilde{A}_{k 1}$ and $x_{2}$ is $\tilde{A}_{k 2}$ and ... and $x_{m}$ is $\tilde{A}_{k m}$ THEN $u_{k 1}$ is $\tilde{B}_{k 1}$ and $u_{k 2}$ is $\tilde{B}_{k 2}$ and ... and $u_{k l}$ is $\tilde{B}_{k l}, \quad k=\overline{1, K}$

where $x_{i}, i=\overline{1, m}$ and $u_{j}, j=\overline{1, l}$ are total input and local output variables , $\tilde{A}_{k i}, \tilde{B}_{k j}$ are fuzzy sets, and $k$ is the number of rules.

The basic steps of the method are given below:

1. The truth degree of the rule is computed as:

$$
r_{j k}=\operatorname{Poss}\left(\tilde{v}_{k} / \tilde{a}_{j k}\right) \cdot c f_{k},
$$

if the sign is "=" and

$$
r_{k}=\left(1-\operatorname{Poss}\left(\tilde{v}_{k} \mid \tilde{a}_{j k}\right)\right) c f_{k},
$$

if the sign is " $\neq$ ". Poss is defined as

$$
\operatorname{Poss}(\tilde{v} \mid \tilde{a})=\max _{u} \min \left(\mu_{\tilde{v}}(u), \mu_{\tilde{a}}(u)\right) \in[0,1] . \tau_{j}=\min \left(r_{j k}\right)
$$

First the objects are evaluated, i.e. every $w_{i}$ object has appropriate linguistic value defined as $\left(v_{i}, c f_{i}\right)$. 
where $v_{i}$ is linguistic value, $\left.\left.c_{f_{k}} \in\right] 0,100\right]$ is confidence degree of the value $v_{i} \cdot v_{k}$ - linguistic value of the rule object, $a_{j k}$ - current linguistic value ( $\mathrm{j}$ is index of the rule, $\mathrm{k}$ is index of relation) value(for example , A_ir)

2. For each rule, calculate

$$
R_{j}=\left(\min _{j} r_{j k}\right) * C F_{j} / 100
$$

where $\mathrm{CF}$ is the confidence degree of the rule.

The user or the creator of the rule defines the firing level $(\pi)$ and $R_{j} \geq \pi$ is checked. If the condition holds true, then the consequent part of rule is calculated.

3.The evaluated $w_{i}$ objects have $S_{i}$ value:

$$
w_{i},\left(v_{i}^{1}, c f_{i}^{1}\right), \ldots, \ldots,\left(v_{i}^{S_{i}}, c f_{i}^{S_{i}}\right)
$$

$S_{i}$ is the number of the rules in fuzzy inference process

The average value is determined as follows:

$$
\bar{v}_{i}=\frac{\sum_{n=1}^{S_{i}} v_{i}^{n} \cdot c f_{i}^{n}}{\sum_{n=1}^{S_{i}} c f_{i}^{n}}
$$

$$
\begin{gathered}
\text { IF } x_{1}=\tilde{a}_{1}^{j} \text { AND } x_{2}=\tilde{a}_{2}^{j} \text { AND ... THEN } y_{1}=\tilde{b}_{1}^{j} \\
\text { AND } y_{2}=\tilde{b}_{2}^{j} \text { AND } \ldots
\end{gathered}
$$

IF $\ldots$ THEN $Y_{1}=A V R G\left(y_{1}\right)$ AND $Y_{2}=A V R G\left(y_{2}\right)$ AND ...

This model has a built-in function AVRG which calculates the average value. This function simplifies the organization of compositional inference with possibility measures. As a possibility measure here a confidence degree is used. So, the compositional relation is given as a set of production rules like:

$$
\begin{gathered}
\text { IF } x_{1}=\tilde{A}_{1}^{j} \text { AND } x_{2}=\tilde{A}_{2}^{j} \text { AND } \ldots \text { THEN } y_{1}=\tilde{B}_{1}^{j} \\
\text { AND } y_{2}=\tilde{B}_{2}^{j} \text { AND },
\end{gathered}
$$

where $j$ is a number of a rule. After all these rules have been executed (with different truth degrees) the next rule (rules) ought to be executed:

$$
\begin{gathered}
\text { IF THEN } Y_{1}=A V R G\left(y_{1}\right) \text { AND } Y_{2}=A V R G\left(y_{2}\right) \\
\operatorname{AND} \ldots
\end{gathered}
$$

Using this model one may construct hypotheses generating and accounting systems. Such system contains the rules:

$$
\text { IF }<\text { condition }_{j}>\text { THEN } X=\tilde{A}_{j} \text { CONFIDENCE } c f_{j}
$$

Here " $X=\tilde{A}_{j}$ " is a hypothesis that the object $X$ takes the value $\tilde{A}_{j}$. Using some preliminary information, this system generates elements $X=\left(\tilde{A}_{j}, R_{j}\right)$, where $R_{j}$ is a truth degree of $j$ th rule. In order to account the hypothesis (i.e. to estimate the truth degree that $X$ takes the value $\tilde{A}_{j}$ ) the recurrent Bayes-Shortliffe formula, generalized for the case of fuzzy hypotheses, is used [3]:

$$
P_{0}=0
$$

$$
P_{j}=P_{j-1}+c f_{j} \operatorname{Poss}\left(\tilde{A}_{0} / \tilde{A}\right)\left(1-\frac{P_{j-1}}{100}\right)
$$

This formula is realized as a built-in function $B S$ :

$$
\text { IF END THEN } P=B S\left(X, \tilde{A}_{0}\right) \text {. }
$$

\section{IV.MODELLING OF DECISION MAKER BY USING POSSIBILITY-PROBABILITY BASED METHOD}

Let us describe the model taking into account the private characteristic features of a decision maker by using the following rules:

\section{Rule 1:}

IF altruism level of decision maker is about 45 and emotion level of decision maker about 40 THEN personal quality of decision maker $(D i)$ is about 35 and $\mathrm{CF}$ is 90

\section{Rule 2:}

IF altruism level of decision maker is about 45 and emotion level of decision maker about 60 THEN personal quality of decision maker $(D i)$ is about 45 and $\mathrm{CF}$ is 55 
Rule 15:

IF altruism level of decision maker is about 65 and emotion level of decision maker about 20

THEN personal quality of decision maker $(D i)$ is about 75 and $\mathrm{CF}$ is 60

It is required to determine the output of the following rule:

IF emotion level of decision maker is about 65 and altruism level of decision maker about 60 THEN personal quality of decision maker $(D i)$ is equal ?

Where the value of linguistic variable are trapezoidal fuzzy numbers. For example,

$$
4 \widetilde{5}=\left\{\begin{array}{l}
\frac{x-30}{12}, 30 \leq x \leq 42 \\
1,42 \leq x \leq 48 \\
\frac{50-x}{2}, 48 \leq x \leq 50 \\
0, \text { otherwise }
\end{array} 6 \tilde{0}=\left\{\begin{array}{l}
\frac{x-50}{5}, 50 \leq x \leq 55 \\
1,55 \leq x \leq 65 \\
\frac{70-x}{5}, 65 \leq x \leq 70 \\
0, \text { otherwise }
\end{array}\right.\right.
$$$$
7 \tilde{5}=\left\{\begin{array}{l}
\frac{x-50}{15}, 50 \leq x \leq 65 \\
1,65 \leq x \leq 80 \\
\frac{85-x}{5}, 80 \leq x \leq 85 \\
0, \text { otherwise }
\end{array}\right.
$$

Dear professor Arkadiy Borisov,

Our department conducts research on decision theory with imperfect information with University of California, Berkeley, during 4 years. One of the branches of our research area is to combine state of nature and state of a decision maker. Consequently, we need to model power of decision maker and features of a decision maker. For the purpose of this we use Delphi Method to collect and to process opinion of experts in decision making area. I would like to ask you as an expert in decision area to express your opinion on main features of a decision maker and a resulting dimension characterizing power of a decision maker (for example, power of a decision maker, personal quality of a decision maker) by participating in our survey. We are sending you $1^{\text {st }}$ questionnaire and I ask you to send back your answers, if possible, within a 3 week.

Kind regards,

R.A.Aliev and L.A. Gardashova

Query 1.

Please indicate by "+" which of the following should be considered as determinants of a decision maker (see in table 1):

Table 1.

\begin{tabular}{|l|l|}
\hline Factor & Mark \\
\hline Trust & \\
\hline Altruism & + \\
\hline Resiprocity & + \\
\hline Emotion & + \\
\hline Risk & + \\
\hline Social responsibility & \\
\hline Tolerance to ambiguity & + \\
\hline Add new factor if necessary & \\
\hline experience & \\
\hline ....... & \\
\hline ........ & \\
\hline
\end{tabular}

Query 2. Identification of total index of a DM.

Please indicate what term should be used for a total index (resulting dimension) of a DM as an overall evaluation to be determined on the base of the determinants indicated in the previous query.
A) personal quality
B) power of decision
C) other(please indicate)

Fig.1. The answers received from experts

The above described model is realized by using the ESPLAN expert system shell and different tests are performed.

Example.
Let us describe the model taking into account the characteristic features of DM:

Rule 1: IF trust level of a DM is about 76 and altruism level of a DM about 45 THEN personal 
quality of a DM (V) is about 46 with confidence degree 0.615 .

Rule 2: IF trust level of a DM is about 35 and altruism level of a DM about 77 THEN personal quality of a DM (V) is about 76 with confidence degree 0.75

It is required: To determine the output (personal quality of a DM),
IF trust level of a DM is about 70 and altruism level of a DM is about 70 THEN personal quality of a $D M(V)$ is equal?

Below is given computer simulation results by ESPLAN expert system shell(see Fig. 2.).
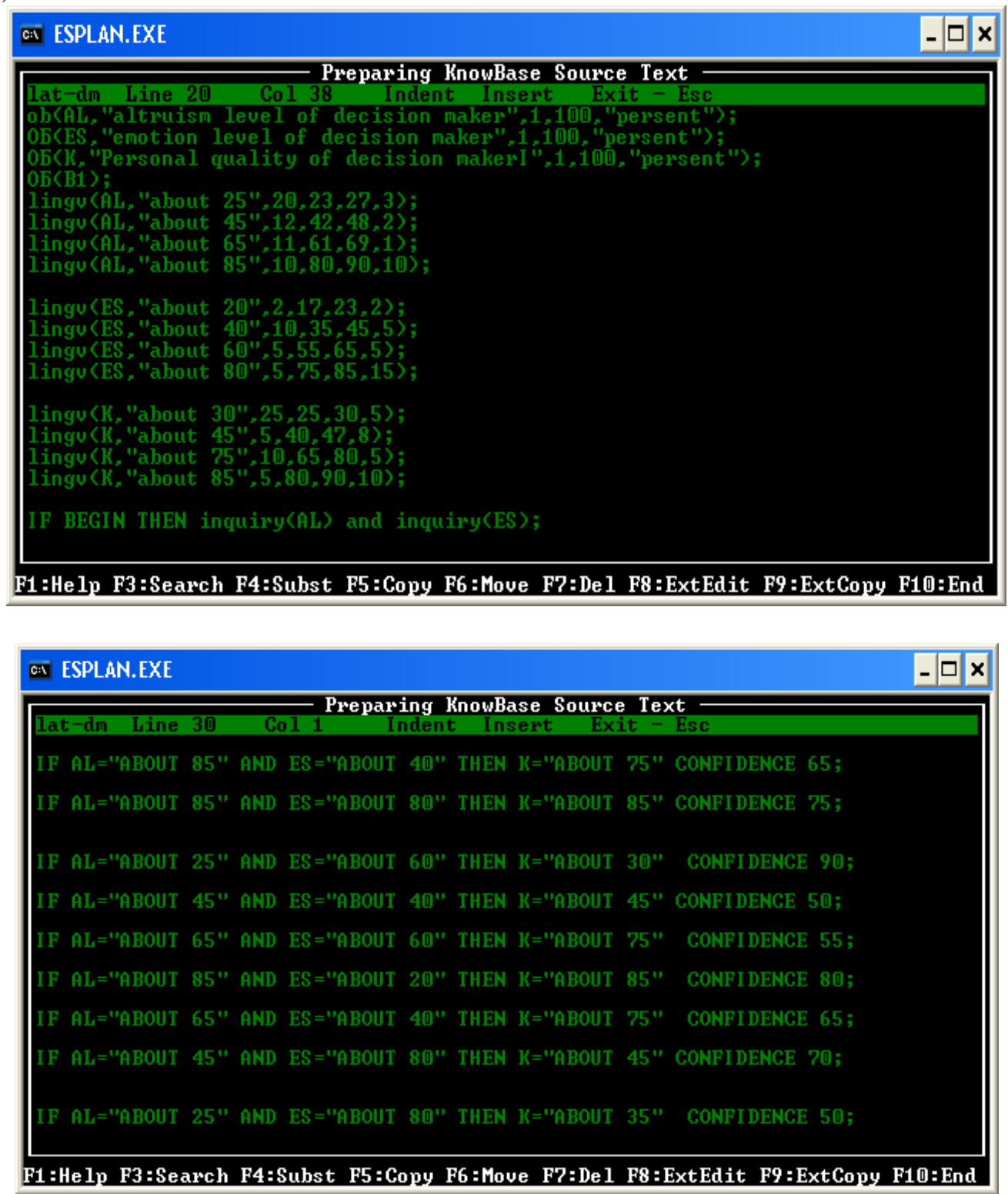

Fig.2. Fragment of computer simulation 

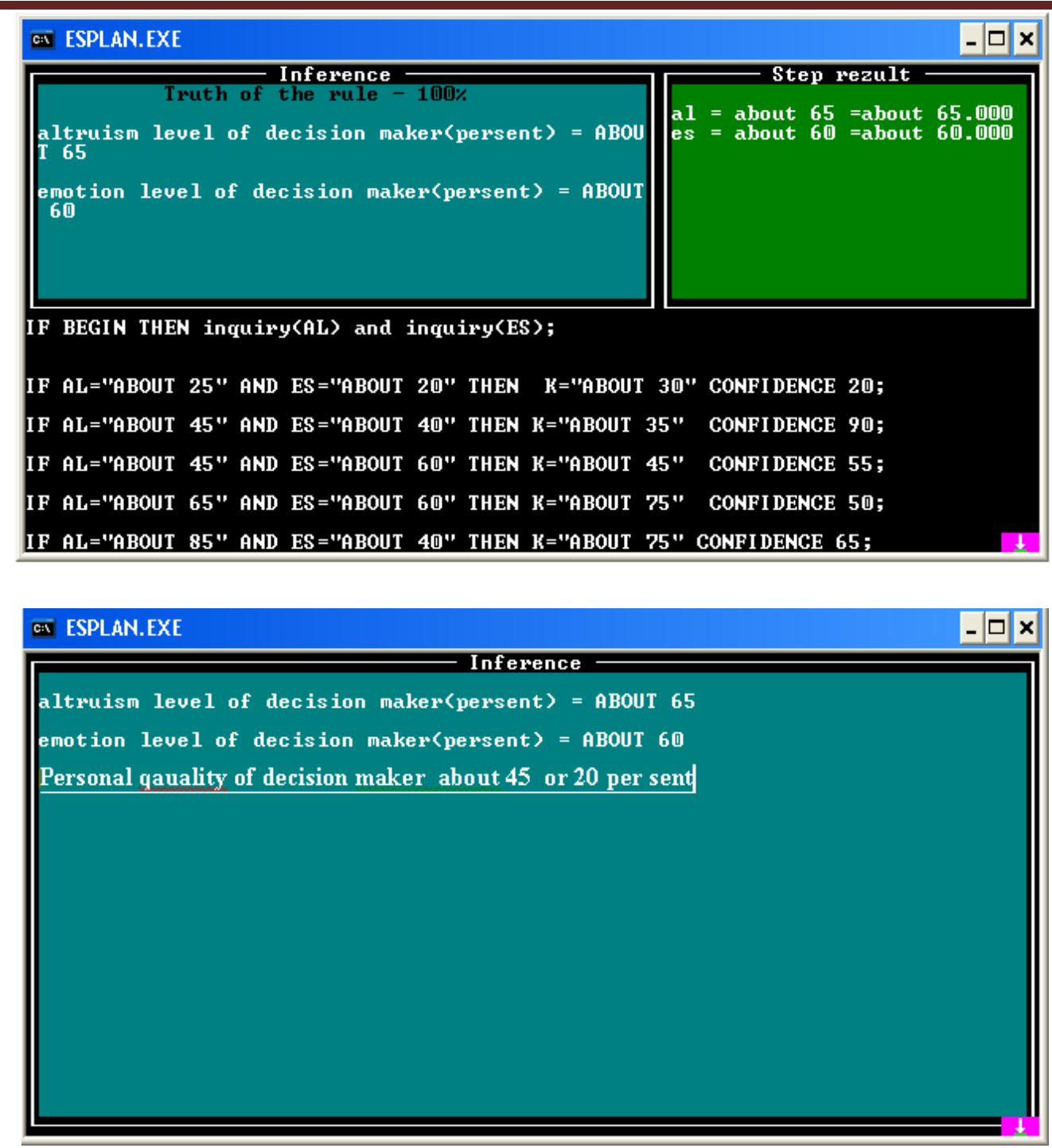

Fig.2. Fragment of computer simulation(continue)

Let us describe step by step calculation results. According possibility-probability based method we are getting[21] :

1.For antecedent part of each rule we obtain:

$$
\begin{aligned}
& r_{11}=\operatorname{Poss}\left(v_{1} / a_{11}\right) \cdot c f_{11}=0.84 \\
& r_{12}=\operatorname{Poss}\left(v_{2} / a_{12}\right) \cdot c f_{12}=0.26 \\
& R_{1}=0.159, \quad \mathrm{R}_{2}=0.21
\end{aligned}
$$

3. Assume that $\pi=0.1$.

4. IF $R_{j} \geq \pi$ is satisfied and each rule is active for fuzzy inference. Defined outputV is between $60.62 \%$ and $70.62 \%$ with confidence degree 0.27

3.The defuzzified value of outputs is defined:64.5
Thus we obtain

IF trust level of a DM is about 70 and altruism level of a DM is about 70 THEN personal quality of a DM $(V)$ is equal about 65 with confidence degree 0.27

For decision making in the given problem provided current characteristic features of decision maker, i.e. the level of altruism and emotion it is possible to calculate personal quality on the basis of the given fuzzy IF-THEN rule. In order to verify the sensitivity of the model the personal quality of decision maker has been investigated under change of level of altruism and emotion.

Described above algorithm is realized by ESPLAN expert system shell. 
The shell of ESPLAN ensures :

- creation of expert systems for various applications;

-building module-oriented structures and segmentation of knowledge bases;

- representation of fuzzy values;

- compositional inference with possibility measures;

- arithmetic operations with fuzzy numbers;

- realization of simple question-ask dialogue by using special functions;

- set a confidence degree for any rule (in per cent);

- call of external programs;

- data interchange using file system.

All above mentioned abilities are supported by ESPLAN knowledge representation language based on production rules.

The inference engine of ESPLAN allows :

-forward-chaining width-first inference with truth degree calculation on the continuous scale $[0,100]$;

- set of a truth threshold during run-time in order to cut a rules with current truth degree less than the threshold;

- tracing inference to the screen;

- tracing inference to disk for further generation of the explanation;

The shell of ESPLAN has own WORDSTAR compatible text editor. The shell of ESPLAN is represented to a user like the multi-window interface.

\section{V.Modelling OF Decision MAKer ON THE BaSiS OF FUZZY AND DEMPSTER-SHAFER THEORY}

Now we consider modeling on the basis of Dempster -Shafer theory. Human behavioral modeling requires an ability to represent and manipulate imprecise cognitive concepts. It also needs to include the uncertainty and unpredictability of human action [17]. Human behavioral modeling requires an ability to formally represent sophisticated cognitive concepts that are often at best described in imprecise linguistic terms. Fuzzy sets provide a powerful tool for enabling the semantical modeling of these imprecise concepts within computer based systems [17]. With the aid of a fuzzy set we can formally represent sophisticated imprecise linguistic concepts in a manner that allows for the types of computational manipulation needed for reasoning in behavioral models based on human cognition and conceptualization.

Now we consider a DM behavioral modeling using fuzzy and Dempster-Shafer theories suggested in [17].

The Dempster-Shafer approach fits nicely into the fuzzy logic since both techniques use sets as their primary data structure and are important components of the emerging field of granular computing. In [17] the behavioral model is represented by partitioning the input space. We can represent relationship between input and output variables by a collection of $\mathrm{n}$ "IF-THEN" rules of the form:

If $X_{1}$ is $\tilde{A}_{i 1}$ and $X_{2}$ is $\tilde{A}_{i 2}, \ldots$ and $X_{r}$ is $\tilde{A}_{i r}$ then $Y$ is $D_{i}$

Here each $\tilde{A}_{i j}$ typically indicates a linguistic term corresponding to a value of its associated variable, furthermore each $\tilde{A}_{i j}$ is formally represented as a fuzzy subset defined over the domain of the associated variable $X_{j}$. Similarly $\tilde{D}_{i}$ is a value associated with the consequent variable $Y$ that is formally defined as a fuzzy subset of the domain of $Y$. To find the output of a DM described by above mentioned rule is used Mamdani inferense method. We consider the consequent to be a fuzzy DempsterShafer granule. Thus we shall now consider the output of each rule to be of the form $Y$ is $m_{i}$ where $m_{i}$ is a belief structure with focal elements $\tilde{D}_{i j}$ which are fuzzy subsets of the universe $Y$ and associated weights $m_{i}\left(\tilde{D}_{i j}\right)$. Thus a typical rule is now of the form

$$
\begin{gathered}
\text { If } X_{1} \text { is } \tilde{A}_{i 1} \text { and } X_{2} \text { is } \tilde{A}_{i 2}, \ldots \text { and } X_{r} \text { is } \tilde{A}_{i r} \\
\text { then } Y \text { is } m_{i}()
\end{gathered}
$$

Using a belief structure to model the consequent of a rule is essentially saying that $m_{i}\left(\tilde{D}_{i j}\right)$ is the probability that the output of the $i^{\text {th }}$ rule lies in the set $\tilde{D}_{i j}$. So rather than being certain as to the output 
set of a rule we have some randomness in the rule. We note that with $m_{i}\left(\tilde{D}_{i j}\right)=1$ for some $\tilde{D}_{i j}$.

Let us describe the reasoning process in this situation with belief structure consequents. Assume the inputs to the system are the values for the antecedent variables, $X_{j}=x_{j}$. For each rule we obtain the firing level,

$$
\tau_{i}=\operatorname{Min}\left[A_{i j}\left(x_{j}\right)\right]
$$

The output of each rule is a belief structure

$$
\hat{m}_{i}=\tau_{i} \wedge m
$$

The focal elements of $\hat{m}_{i}$ are $\tilde{F}_{i j}$, a fuzzy subset of $Y$ where $F_{i j}(y)=\operatorname{Min}\left[\tau_{i}, D_{i j}(y)\right]$, here $\tilde{D}_{i j}$ is a focal element of $m_{i}$. The weights associated with these new focal elements are simply $\hat{m}_{i}\left(\tilde{F}_{i j}\right)=\hat{m}_{i}\left(\tilde{D}_{i j}\right)$.

The overall output of the system $\mathrm{m}$ is obtained by taking a union of the individual rule outputs,

$$
m=\bigcup_{i=1}^{n} \hat{m}_{i}
$$

For every a collection $<\tilde{F}_{1 j_{1}}, \ldots \tilde{F}_{n j_{1}}>$ where $\tilde{F}_{i j_{1}}$ is a focal element of $m_{i}$ we obtain a focal element of $m, \tilde{E}=\bigcup_{i} \tilde{F}_{i j_{1}}$ and the associated weight is

$$
m(\tilde{E})=\prod_{i=1}^{n} \hat{m}_{i}\left(\tilde{F}_{i j_{1}}\right)
$$

As a result of this third step it is obtained a fuzzy $\mathrm{D}-\mathrm{S}$ belief structure $V$ is $\mathrm{m}$ as output of the agent.

We denote the focal elements of $m$ as the fuzzy subsets $\tilde{E}_{j}, j=1$ to $q$, with weights $m\left(\tilde{E}_{j}\right)$.

Let us describe the model taking into account the characteristic features of DM. DM's behavioral model can be described as[21]:

Rule 1: IF trust level of a DM is about 76 and altruism level of a DM about 45 THEN personal quality of a DM (V) is $m_{1}$.
Rule 2: IF trust level of a DM is about 35 and altruism level of a DM about 77 THEN personal quality of a DM (V) is $m_{2}$.

Let us determine the output (personal quality of a $\mathrm{DM}$ ), if trust level of a DM is about 70 and altruism level of a DM is about 70: $m_{1}$ has focal elements $\quad \tilde{D}_{11}=4 \tilde{6} \quad$ with $\quad m\left(\tilde{D}_{11}\right)=0.7 \quad$ and $\tilde{D}_{12}=4 \tilde{8} \quad$ with $m\left(\tilde{D}_{11}\right)=0.3, m_{2}$ has focal elements $\quad \tilde{D}_{21}=7 \tilde{6} \quad$ with $\quad m\left(\tilde{D}_{21}\right)=0.2 \quad$ and $\tilde{D}_{22}=\tilde{8} 1$ with $m\left(\tilde{D}_{22}\right)=0.8$

The values of linguistic variables are trapezoidal fuzzy numbers:

$$
\begin{aligned}
4 \tilde{6} & =\left\{\begin{array}{l}
\frac{x-40}{6}, 40 \leq x \leq 46 \\
1, x=46 \\
\frac{65-x}{19}, 46 \leq x \leq 65 \\
0, \text { otherwise }
\end{array}\right. \\
4 \tilde{8} & =\left\{\begin{array}{l}
\frac{x-40}{8}, 40 \leq x \leq 48 \\
1, x=48 \\
\frac{65-x}{17}, 48 \leq x \leq 65 \\
0, \text { otherwise }
\end{array}\right. \\
7 \tilde{6} & =\left\{\begin{array}{l}
\frac{x-61}{15}, 61 \leq x \leq 76 \\
1, x=76 \\
\frac{95-x}{19}, 76 \leq x \leq 95 \\
0, \text { otherwise }
\end{array}\right. \\
8 \tilde{1} & =\left\{\begin{array}{l}
\frac{x-61}{20}, 61 \leq x \leq 81 \\
1, x=81 \\
\frac{95-x}{14}, 81 \leq x \leq 95 \\
0, \text { otherwise }
\end{array}\right.
\end{aligned}
$$

Let us calculate the belief values for each rule. By using [17] in this example the empty set takes the value 0.09. But in accordance with Dempster-Shafer theory m-value of the empty set should be zero. In order to achieve this, $m$ values of the focal elements should be normalized and $m$ value of the empty set 
made equal to zero. The normalization process is as follows:

1) Determine $T=\sum_{A_{i} \cap B_{i}=\varnothing} m_{1}\left(\tilde{A}_{i}\right) \cdot m_{2}\left(\tilde{B}_{i}\right)$

2) For all $\tilde{A}_{i} \cap \tilde{B}_{i}=\varnothing$ weights

$m\left(\tilde{E}_{k}\right)=\frac{1}{1-T} m_{1}\left(\tilde{A}_{i}\right) \cdot m_{2}\left(\tilde{B}_{j}\right)$

3) For all $\tilde{E}_{k}=\varnothing$ sets $m\left(\tilde{E}_{k}\right)=0$

In accordance with the procedures described above:

$$
\begin{gathered}
m_{3}=(\{4 \tilde{6}\})=0.230769, \\
m_{3}=(\{4 \tilde{6}, y\})=0.384615, \\
m_{3}=(\{4 \tilde{0}, 4 \tilde{5}\})=0.230769 \\
m_{3}=(\{4 \tilde{0}, 4 \tilde{5}\})=0.230769 \\
\operatorname{Bel}(\{4 \tilde{6}, y\})=0.615385 .
\end{gathered}
$$

For the second rule: $\operatorname{Bel}(\{7 \tilde{6}, y\})=0.753425$. Firing level of the $i$-th rule is equal to the minimum among all degrees of membership of a system input to antecedent fuzzy sets of this rule:

$$
\tau_{i}=\min _{j=1}^{n}\left[\max _{X_{j}}\left(A^{\prime}\left(x_{j}\right) \wedge A_{i j}\left(x_{j}\right)\right)\right] .
$$

For example, over first rule we are getting:

$\tau_{1}=\min [\max (\{0 / 51,0.68 / 70,1 / 75,1 / 83,0 / 84\},(\{0 / 51$,

$1 / 70,0.74 / 75,0.31 / 83,0.26 / 84,0 / 89))), \max (\{1 / 51,1 / 55,0.8 / 56$,

$0 / 61\} \cap\{0 / 51,0.21 / 55,0.26 / 56,0.5 / 61\}))=\min (0.74,0.26)=0.26$

The firing levels of each rule are $\tau_{1}=0.26$ and $\tau_{2}=0.28$.

The output of each rule is a belief structure:

$$
\begin{aligned}
& \tilde{m}_{1}=\tau_{1} \wedge \tilde{m}, \quad \tilde{m}_{2}=\tau_{2} \wedge \tilde{m} \\
& F_{11}=\tau_{1} \wedge D_{11}=\left\{\frac{0.26}{42}, \frac{0.26}{46}, \frac{0.26}{50}\right\}, m_{1}\left(F_{11}\right)=0.7 \\
& F_{12}=\tau_{1} \wedge D_{12}=\left\{\frac{0.26}{44}, \frac{0.26}{50}, \frac{0.26}{100}\right\}, m_{1}\left(F_{12}\right)=0.3
\end{aligned}
$$

$$
\begin{aligned}
& F_{21}=\tau_{2} \wedge D_{21}=\left\{\frac{0.28}{69}, \frac{0.28}{76}, \frac{0.28}{83}\right\}, m_{2}\left(F_{21}\right)=0.2 \\
& F_{22}=\tau_{2} \wedge D_{22}=\left\{\frac{0.28}{74}, \frac{0.28}{81}, \frac{0.28}{100}\right\}, m_{2}\left(F_{22}\right)=0.8
\end{aligned}
$$

We obtain a focal element of $m, \tilde{E}=\bigcup_{i} \tilde{F}_{i j_{1}}$ and the associated weight:

$$
\begin{aligned}
& m=\cup_{i=1} \cup \tilde{m}_{i}, \mathrm{n}=2 \\
& E_{1}=F_{11} \cup F_{21}=\left\{\frac{0.26}{42}, \frac{0.26}{46}, \frac{0.26}{50}, \frac{0.28}{69}, \frac{0.28}{76}, \frac{0.28}{83}\right\}, \\
& m\left(E_{1}\right)=0.7 \cdot 0.2=0.14 \\
& E_{2}=F_{11} \cup F_{22}=\left\{\frac{0.26}{42}, \frac{0.26}{46}, \frac{0.26}{50}, \frac{0.28}{74}, \frac{0.28}{81}, \frac{0.28}{100}\right\}, \\
& m\left(E_{2}\right)=0.7 \cdot 0.8=0.56 \\
& E_{3}=F_{12} \cup F_{21}=\left\{\frac{0.26}{44}, \frac{0.26}{50}, \frac{0.26}{100}, \frac{0.28}{69}, \frac{0.28}{76}, \frac{0.28}{83}\right\}, \\
& m\left(E_{3}\right)=0.3 \cdot 0.2=0.06 \\
& E_{4}=F_{12} \cup F_{22}=\left\{\frac{0.26}{44}, \frac{0.26}{50}, \frac{0.26}{100}, \frac{0.28}{74}, \frac{0.28}{81}, \frac{0.28}{100}\right\}, \\
& m\left(E_{4}\right)=0.3 \cdot 0.8=0.24
\end{aligned}
$$

The defuzzified values of focal elements obtained by using the center of gravity method are the following:

$$
\begin{aligned}
& \operatorname{Defuz}\left(E_{1}\right)=\bar{y}_{1}=61.55556 ; \\
& \operatorname{Defuz}\left(E_{2}\right)=\bar{y}_{2}=64.14815 ; \\
& \operatorname{Defuz}\left(E_{3}\right)=\bar{y}_{3}=62.51852 ; \\
& \operatorname{Defuz}\left(E_{4}\right)=\bar{y}_{4}=65.11111
\end{aligned}
$$

$\bar{y}=m\left(E_{1}\right) \cdot \operatorname{def}\left(E_{1}\right)+m\left(E_{2}\right) \cdot \operatorname{def}\left(E_{2}\right)+$

$+m\left(E_{3}\right) \cdot \operatorname{def}\left(E_{3}\right)+m\left(E_{4}\right) \cdot \operatorname{def}\left(E_{4}\right)$

The defuzzified value of $m$ is $\bar{y}=63.92$.

Thus we determine the rule with the output as:

IF trust level of a DM is about 70 and altruism level of a DM about 70 THEN personal quality of a decision maker $(V)$ is equal to 63.92 percent.

In this work the decision maker behavioral modelling under imperfect information is discussed. Above described methods of fuzzy inference have 
been analyzed, for each of methods a number of test were performed, which demonstrated the approximately same results. Both methods are efficient, but the preference of the second method is the possibility to evaluate the confidence degree of the result.

\section{CONCLUSION}

In this paper, the decision maker behavioral modelling under imperfect information or secondorder uncertainty is proposed. By using Delphi method psychological determinants of a decision maker were determined. The described models are realized by using the expert system shell, the language of technical computing Matlab and different tests are performed. The obtained results proved validity of the suggested approach.

\section{REFERENCES}

[1]L.A. Zadeh, "Computing with words and perceptions - a paradigm shift", In: Proceedings of the IEEE International Conference on Information Reuse and Integration, Las Vegas (Nevada, USA: IEEE Press ), 2009, pp. 450-452.

[2] http://superemotional.blogspot.com/2010/01/ biography-of-prof-lotfi-zadeh.html

[3]R.A.Aliyev R.A.,D. E. Gulko, M. M. Shakhnazarov, "Expert system for production planning" J.News of Academy of Sciences of the USSR,Tekhn. Cybernetics,No.5,1988, pp. 25-30.

[4]J.C. Cox, D.Friedman ., V. Sadiraj V. "Revealed altruism",J.Econometrica,Vol.6, 2008,pp.:31-69.

[5]J.C.Cox, "How to identify trust and reciprocity", J.Games and Economic Behavior, 46, 2004, pp. 260-281.

[6]J.C.Cox , D.Friedman , S.Gjerstad, "A tractable model of reciprocity and fairness", $J$. Games and Economic Behavior, 59, 2007 , pp.17-45.

[7]J.C.Cox , K.Sadiraj, V.Sadiraj, "Implications of trust, fear, and reciprocity for modeling economic behavior". Exp.. Econ, 11(1), 2008, pp.1-24.

[8] J.C.Cox , E.Ostrom , J.M.Walker, A.J.Castillo , E.Coleman , R.Holahan , M.Schoon , B.Steed, "Trust in Privative and Common Property Experiments", Southern Economic Journal, 75(4),2009, pp.957-975.
[9]K.Abbink , B.Irlenbusch, E. Renner, "The Moonlighting game: An empirical Study on Reciprocity and Retribution", Journal of Economic Behavior and Organization, 42, 2000,pp.265-277.

[10]J.Andreoni , J.Miller, "Giving according to GARP: An experimental Test of the Consistency of Preferences for Altruism", Econometrica, 70, 2002, pp.737-753.

[11]J.Berg ， J.Dickhaut ， K.Mccabe, "Trust, reciprocity, and Social History", J.Games and Economic Behavior, 10, 1995 pp.122-142.

[12]A.Falk , U.Fischbacher, "A Theory of Reciprocity", J. Games and Economic Behavior, 54, 2006, pp.122-142.

[13]R.Bosman , F.Van Winden F, "Emotional Hazard in a Power-to-take Experiment" Economic Journal, 112, 2002, pp.147-169.

[14]E.Fehr, K.M.Schmidt, "A Theory of fairness, Competition, and Cooperation", Quarterly Journal of Econometrics, 114, 1999, pp.817-868.

[15] D.Kahneman, A.Tversky, "Prospect theory: an analysis of decision under uncertainty", Econometrica, 47(2), 1979, pp.263-291.

[16] L.A. Gardashova, "Economic agents behavior modeling under second-order uncertainty" In: Proceedings Ninth international conference on Application of Fuzzy Systems and Soft computing, Prague, Czech Republic, 2010, pp.359-364.

[17] R.R.Yager, "Human behavioral modeling using fuzzy and Dempster-Shafer theory", In H Liu, JJ Salerno \& MJ Young (Eds.) Social computing, behavioral modeling, and prediction.New York: Springer.,2008, pp. 89-99.

[18]R.R.Yager, "Using knowledge trees for semantic web querying, in Fuzzy Logic and the Semantic Web", edited by Sanchez E, Elsevier: Amsterdam.2006, pp. 231-246.

[19] L.A. Gardashova, "A new approach to solving decision making problem with Z-information under uncertain environment", 2nd World Conference on Soft Computing, 2012, Baku, 2012, pp.464-470.

[20] L.A. Gardashova "Application of operational approaches to solving decision making problem using Z-numbers". // journal, Applied Mathematics, USA, 2014, Vol.5, No 9,pp.13231334 J.Applied Mathematics., in press. 
[21]L.A.Gardashova. Decision making in management systems of oil refinery enterprise under fuzzy environment. Doctoral thesis, Baku, 2012,-308 p.

[22] A Falk, U.Fischbacher. A Theory of Reciprocity, Cames and Economic Behavior,54,2006,pp.293315

[23]J.C.Cox, D. Friedman, V. Sadiraj. Revealed altrism. Econometrica, Vol.6, No.1, 2008, pp. 3169.

[24] J.C.Cox. How to identify trust and reciprocity. Cames and Economic Behavior, 46, 2004, pp.260-281.

[25] J.C.Cox, D. Friedman, S. Gjerstad. A tractable model of reciprocity and fairness. Cames and Economic Behavior, 59, 2007, pp. 17-45.

[26] J.C.Cox, K. Sadiraj, V. Sadiraj. Implications of trust, fear, and reciprocity for modeling economic behavior. ExpEcon., 11,2008, pp.1-24.

[27] J.C.Cox, E.Ostrom, J.M.Walker,A.J,Castillo, E. Coleman, R. Holahan, M. Schoon, and B. Steed, Trust in Private and Common Property Experiments.Southern Economic Journall,2009, 75(4), pp.957-975.

[28]K. Abbink, B.Irlenbusch and E. Renner. The Moonlighting Game: An Empirical Study on Reciprocity and Retribution.Journal of Economic Behavior and Organization,42, 2000, pp.265-277.

[29]J. Andreoni,J.Miller. Giving According to GARP:An Experimental Test of the Consistency of Preferences for Altruism. Econometrica, 70, 2002, pp. 737-753

[30] J. Berg, J. Dickhaut, K. Mccabe. Trust, Reciprocity, and Social History. Cames and Economic Behavior,10, 1995, pp.122-142

[31] L.A.Gardashova., E.H.Aliyev. A Fuzzy approach to evaluation of democracy index. International journal of Business, Humanities and Technology, USA, Vol 4.,No 5, 2014, pp.94-98 